\title{
Automatic Segmentation and Components Classification of Optic Pathway Gliomas in MRI
}

\author{
Lior Weizman ${ }^{1}$, Liat Ben-Sira ${ }^{2}$, Leo Joskowicz ${ }^{1}$, Ronit Precel $^{2}$, \\ Shlomi Constantini ${ }^{2}$, and Dafna Ben-Bashat ${ }^{2}$ \\ 1 School of Eng. and Computer Science, Hebrew University of Jerusalem, Israel \\ 2 Sourasky Medical Center, Tel-Aviv, Israel \\ lweizm45@cs.huji.ac.il
}

\begin{abstract}
We present a new method for the automatic segmentation and components classification of brain Optic Pathway Gliomas (OPGs) from multi-spectral MRI datasets. Our method accurately identifies the sharp OPG boundaries and consistently delineates the missing contours by effectively incorporating prior location, shape, and intensity information. It then classifies the segmented OPG volume into its three main components - solid, enhancing, and cyst - with a probabilistic tumor tissue model generated from training datasets that accounts for the datasets grey-level differences. Experimental results on 25 datasets yield a mean OPG boundary surface distance error of $0.73 \mathrm{~mm}$ and mean volume overlap difference of $30.6 \%$ as compared to manual segmentation by an expert radiologist. A follow-up patient study shows high correlation between the clinical tumor progression evaluation and the component classification results. To the best of our knowledge, ours is the first method for automatic OPG segmentation and component classification that may support quantitative disease progression and treatment efficacy evaluation.
\end{abstract}

\section{Introduction}

Optic Pathway Gliomas (OPGs) are the most common brain tumors of the central nervous system in patients with Neurofibromatosis (NF) 1]. OPGs are low-grade pilocytic astrocytomas that arise in the optic nerve and chiasm and may involve the hypothalamus and post-chiasmal regions. OPGs may be asymptomatic, but may become very aggressive and cause severe complications depending on their location [2]. Patients with known OPGs are typically screened serially for progressive visual loss and for changes on MR images. Precise follow-up of an OPG requires the quantification of the tumor volume and the classification of its components into solid, enhancing, and cyst regions. Evolution or changes in the tumor volume and its components may serve as markers for disease progression and may be used to determine the proper treatment and to evaluate its efficacy. Therefore, the accurate quantification of the tumor volume and identification of its components is crucial [3. Currently, OPG volume is coarsely estimated manually by the physician with a few measurements on axial, coronal, and sagittal slices. This is inaccurate, time consuming, error prone, user dependent, and may compromise the follow-up of the disease progression and its treatment.

T. Jiang et al. (Eds.): MICCAI 2010, Part I, LNCS 6361, pp. 103-110, 2010.

(C) Springer-Verlag Berlin Heidelberg 2010 
Brain tumor detection, characterization, and follow-up based on CT and MR images is currently the standard of care in radiology. The ample spectrum of tumor types and locations has given rise to a plethora of methods for tissue classification and quantification. Most studies focus on the automatic detection of Glioblastoma Multiforme (GBM) tumors 345, as they account for $40 \%$ of all primary malignant brain tumors in adults [6]. Additional studies address of other brain lesions, e.g. astrocytoma [7] and low-grade glioma [8].

While effective, most methods do not take into account the anatomic location of the tumor, which is key for the detection and segmentation of OPGs. A common problem of OPGs and other tumors is the delineation of their boundaries due to the tumor inhomogeneity, the surrounding tissues with overlapping image intensity values, the uneven tumor ingrowth into nearby structures, and the imaging partial volume effect. In addition, most existing automatic tumor components classification methods are based on learning the grey-level range of every component from a training set [4]. Therefore, they might suffer from sensitivity to grey-level differences between the learning and the testing sets.

In this paper we describe a new automatic method for the segmentation and components classification of OPG from multi-spectral MRI datasets. Our method effectively incorporates prior location, shape, and intensity information to accurately identify the sharp OPG boundaries and to consistently delineate the OPG contours that cannot be clearly identified on standard MR images. It then classifies the segmented OPG volume into its solid, enhancing, and cyst components based on a probabilistic tumor tissue model generated from training datasets that overcomes the grey-level differences between the learning and the test datasets. Our experimental study on 25 datasets yields a mean surface distance error of $0.73 \mathrm{~mm}$ and a mean volume overlap difference of $30.6 \%$ as compared to manual segmentation by an expert radiologist. A follow-up study shows high correlation between the clinical tumor progression evaluation and the component classification results. The advantages of our method are that it is automatic, accurate, consistent, and that it may support quantitative disease progression, treatment decision-making, and treatment efficacy evaluation.

\section{OPG Segmentation and Classification}

Our method inputs the patient multi-spectral MRI datasets, which include T1weighted, T2-weighted, and Fluid Attenuated Inversion Recovery (FLAIR) pulse sequences, and a prior OPG spatial location. The OPG prior spatial location consists of the OPG Region Of Interest (ROI) $M$, and the chiasm core $O$, both defined by an expert radiologist on an anatomy atlas. The output is the OPG boundary and OPG voxel classification into solid, enhancing, and cyst components.

The method proceeds in four steps. First, the multi-spectral MR images are coregistered, normalized for intensity, and registered to the anatomy atlas to detect prior OPG ROI and chiasm core. Next, the OPG sharp boundaries are found. In the third step, the missing OPG boundary segments are computed from a probabilistic tumor tissue model generated from training datasets. Finally, the OPG voxels are classified into solid, enhancing, and cyst components. 


\subsection{MR Images Coregistration and Normalization}

Since the patient may move during image acquisition, we first coregister the MR images with the SPM affine registration method 9. We then standardize the patient MRI intensity values and the probabilistic OPG intensity model with the Dynamic Histogram Warping method [10. The OPG ROI and the chiasm core are identified in the resulting intensity normalized and aligned patient MR images by registering them to the labeled anatomy atlas with the SPM normalization method 9]. The OPG ROI $M=\left\{m_{1}, \ldots, m_{n_{M}}\right\}$ and chiasm core $O=\left\{o_{1}, \ldots, o_{n_{O}}\right\}$ point sets are then mapped back from the prior atlas space to the patient image space. The resulting sets $\tilde{M}=\left\{\tilde{m}_{1}, \ldots, \tilde{m}_{n_{\tilde{M}}}\right\}$ and $\tilde{O}=\left\{\tilde{o}_{1}, \ldots, \tilde{o}_{n_{\tilde{O}}}\right\}$ represent the chiasm core and the OPG ROI in the patient image space.

\subsection{OPG Sharp Boundaries Detection}

The OPG is mostly surrounded by the Cerebral Spine Fluid (CSF), whose intensity value in the FLAIR pulse sequence is very low. Thus, the OPG sharp boundaries are clearly distinguishable where the CSF surrounds the OPG. The CSF voxels are identified in FLAIR by fixed-value thresholding. The sharp OPG boundary voxels are identified as follows. For every voxel $\tilde{m}_{i} \in \tilde{M}$, we find the shortest Euclidean distance path to $\tilde{O}$ and label it as $P_{i}=\left\{p_{1}, \ldots, p_{l}\right\}$. If at least one of the voxels in $P_{i}$ is a CSF voxel, then $\tilde{m}_{i}$ is removed from $\tilde{M}$. The resulting $\tilde{M}$ does not contains the voxels in the OPG ROI that lie beyond the CSF borders surrounding the OPG. This step enforces a convex shape, which is mostly the case in OPG. Fig. 1 illustrates this step.

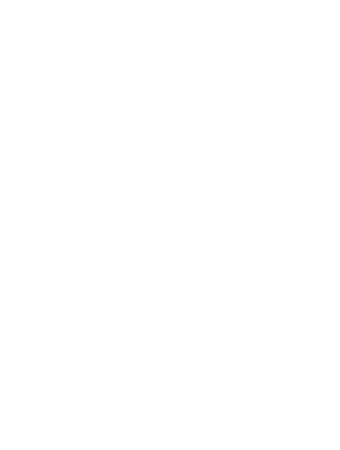

(a)

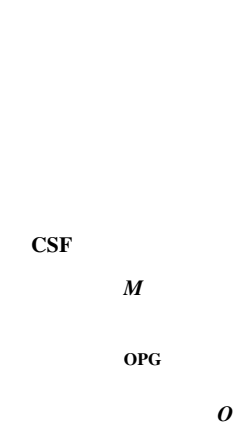

(b)

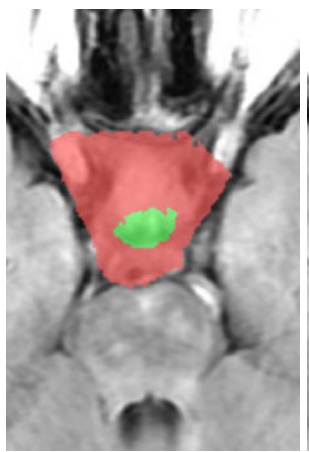

(c)

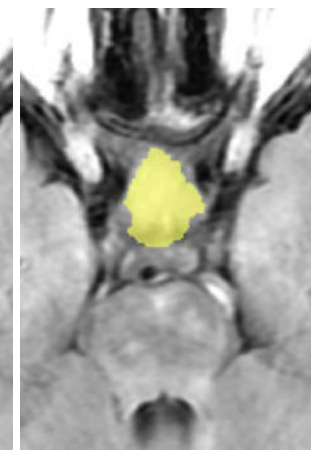

(d)

Fig. 1. (a) OPG location in the brain; (b) OPG ROI $\tilde{M}$ (red), chiasm core $\tilde{O}$ (green), OPG (yellow), CSF (blue) areas; (c) example of the OPG ROI (red) and chiasm core (green) on a sample slice; (d) sharp boundary detection result (yellow) 


\subsection{OPG Boundary Completion}

To find the missing OPG boundary segments where a clear border with CSF does not exist, we use the Generalized Likelihood Ratio Test (GLRT) 11. We define two complementary hypotheses - healthy tissue and OPG tissue - and choose between them based a probabilistic measure computed from an estimate of their unknown model parameters. We describe these two steps in detail next.

Probabilistic tissue model. We represent the multi-spectral MRI dataset consisting of $k$ pulse sequences, each with $n$ voxels, as a set $V=\left\{v_{1}(r), \ldots, v_{n}(r)\right\}$ where $v_{i}(r)$ is a $k$-dimensional vector, and $v_{i}(r)=\left(v_{i}^{1}, v_{i}^{2}, \ldots, v_{i}^{j}, \ldots, v_{i}^{k}\right)$, where $v_{i}^{j}$ represents the intensity value of the voxel $v_{i}$ in the $j$-th pulse sequence. The parameter $r$ denotes the spatial location of the voxel $v_{i}(r)$.

We postulate two hypotheses for voxel $v_{j}(r)$ :

$H_{0}$ : voxel $v_{j}(r)$ corresponds to healthy tissue.

$H_{1}$ : voxel $v_{j}(r)$ corresponds to OPG tissue.

The probability of $v_{j}(r)$ to be OPG tissue depends on its spatial location and on the voxel intensity values in the MR images.

Since the every voxel in the image can have any intensity level, the spatial location of a voxel can be assumed to be independent of its intensity level. Therefore, the Probability Density Function (PDF) of $v_{j}(r)$ for a given hypothesis is:

$$
f\left(v_{j}(r), r \mid H_{i}\right)=f_{I}\left(v_{j}(r) \mid H_{i}\right) \cdot f_{S}\left(r \mid H_{i}\right), i=0,1
$$

where $f_{I}\left(v_{j}(r) \mid H_{i}\right)$ and $f_{S}\left(r \mid H_{i}\right)$ are the respective intensity and spatial location contributions to $f\left(v_{j}(r), r \mid H_{i}\right)$.

Since the OPG spreads from the center of the core to the margins of the chiasm, we model $f_{S}\left(r \mid H_{1}\right)$ as a Gaussian, with mean $r_{S}$ and covariance matrix $\mathbf{C}_{S}$. Since $H_{0}$ is the complementary hypothesis of $H_{1}$, we obtain:

$$
f_{S}\left(r \mid H_{0}\right)=1-f_{S}\left(r \mid H_{1}\right)
$$

We model the intensity value of healthy/OPG voxels as a mixture of Gaussians:

$f_{I}\left(v_{j}(r) \mid H_{i}\right)=\sum_{q=1}^{3} a_{i q} \cdot \frac{1}{(2 \pi)^{k / 2}\left|\mathbf{C}_{i q}\right|^{1 / 2}} \exp \left\{-\frac{1}{2}\left(v_{j}(r)-\mu_{i q}\right)^{T} \mathbf{C}_{i q}^{-1}\left(v_{j}(r)-\mu_{i q}\right)\right\}$

where the superscript $T$ denotes the matrix transpose. The parameters $\left\{\mu_{0 q}\right\}_{q=1}^{3}$ and $\left\{\mathbf{C}_{0 q}\right\}_{q=1}^{3}$ denote the mean vector and covariance matrix of the healthy tissue component: air, CSF, and non-enhancing healthy tissue, respectively. The parameters $\left\{\mu_{0 q}\right\}_{q=1}^{3}$ and $\left\{\mathbf{C}_{1 q}\right\}_{q=1}^{3}$ denote the mean vector and covariance matrix of solid, enhancing, and cyst OPG components, respectively. Since we do not have the prior probabilities for these components for either the healthy or the OPG hypothesis, we set them to have equal prior probability, i.e. $\forall i, q a_{i q}=\frac{1}{3}$. 
Unknown parameters estimation. The Maximum Likelihood Estimators (MLEs) of the unknown model parameters, given the training data, are as follows [11. The parameters $\left\{\hat{\mu}_{0 q}\right\}_{q=1}^{3}$ and $\left\{\hat{\mathbf{C}}_{0 q}\right\}_{q=1}^{3}$ are the sample mean and covariance matrix of the CSF, air, and healthy non-enhancing tissue components of healthy tissues, respectively. Similarly, $\left\{\hat{\mu}_{1 q}\right\}_{q=1}^{3}$ and $\left\{\hat{\mathbf{C}}_{1 q}\right\}_{q=1}^{3}$ are the sample mean and covariance of solid, enhancing, and cystic components of OPG, respectively. The parameters $\hat{r}_{S}$ and $\hat{\mathbf{C}}_{s}$ are the center of mass and the spatial sample covariance matrix of $\tilde{O}$. The GLRT is thus:

$$
\Lambda\left(v_{j}(r)\right)=\frac{f\left(v_{j}(r), r \mid \hat{\theta_{1}}, \hat{\theta_{2}} ; H_{1}\right)}{f\left(v_{j}(r), r \mid \hat{\theta_{0}}, \hat{\theta_{2}} ; H_{0}\right)} \underset{H_{0}}{\gtrless} \gamma
$$

where $\gamma$ is a predetermined threshold that reflects the trade-off between false and missed detections. The notation $\underset{H_{0}}{\stackrel{H_{1}}{\gtrless}}$ means that if $\Lambda\left(s_{i}(r)\right)$ is greater than $\gamma, H_{1}$ is chosen for voxel $s_{i}(r)$, otherwise, $H_{0}$. The final segmentation result is the intersection between the GLRT result and $\tilde{M}$. The set of voxels $S=\left\{s_{i}(r)\right\}$ that are detected as OPG is thus:

$$
S=\left\{s_{i}(r): \Lambda\left(s_{i}(r)\right)>\gamma \text { and } s_{i}(r) \in \tilde{M}\right\}
$$

\subsection{OPG Internal Classification}

A common problem of the state-of-the-art supervised classification methods is that the classification results are affected by different acquisition parameters of the training and testing datasets. We propose to use a classification technique that overcomes this phenomenon when the training and the testing datasets intensities differ by a multiplicative factor, as is a common case in OPG datasets.

To determine if a given OPG voxel is solid, enhancing, or cyst, we use the Spectral Angle Mapper (SAM) method [12. SAM classification is based on the angle measured between the given vector of pulse sequences grey-levels and a training vector previously computed for every OPG component. To classify a given set of OPG voxels, $S=\left\{s_{i}(r)\right\}_{i=1}^{N_{S}}$, we use the estimations of the solid, enhancing, and cystic components, $\hat{\mu}_{11}, \hat{\mu}_{12}, \hat{\mu}_{13}$, which were previously calculated. Following the SAM approach, the angle between $s_{i}(r)$ and $\hat{\mu}_{1 q}$ is: $\varphi_{q}=\operatorname{acos}\left(s_{i}(r) \cdot \hat{\mu}_{1 q}\right)$, where - denotes the vector dot product. Consequently, $s_{i}(r)$ is assigned to the component represented by $\hat{\mu}_{1 q}$ that yields the lowest $\varphi_{q}$ for $q=1,2,3$.

\section{Experimental Results}

We conducted a quantitative evaluation of our method with clinical multispectral MRI datasets of 7 pediatric patients, 3-7 years old with OPGs. The patients were serially screened every several months to produce a total of 28 datasets. The MR images were acquired by General Electric Signa 1.5T HD. The study was approved by the local ethical research committee. Each scan consists of T1-weighted, T2-weighted, and FLAIR. Each dataset has $512 \times 512 \times 30$ 
voxels with voxel size $0.5 \times 0.5 \times 5.0 \mathrm{~mm}^{3}$. An expert radiologist defined the prior spatial inputs, $O$ and $M$, on the Johns Hopkins University International Consortium of Brain Mapping T2 atlas [13, and manually produced ground-truth classified segmentations for each scan. A second expert radiologist reviewed and revised the segmentations. To separate the training and testing datasets and to provide robust performance of our methods, three data sets were used to estimate the unknown parameters of the model and to determine the CSF value in the FLAIR sequence to distinguish the OPG from CSF in their tangency region. The remaining 25 scans were used to evaluate the proposed method. All the results were obtained with an experimentally determined threshold of $\gamma=1.2$.

In the first study, we applied the OPG segmentation algorithm (Secs 2.12.3 ) to each of the 25 cases. Fig. 2 shows the segmentation results in three common validation measures [16. The average symmetric surface distance is $0.73 \mathrm{~mm}$, and the volumetric overlap error is $30.6 \%$. These values are comparable to those of other automatic detection methods of brain tumors reported in the literature [45], and to the inter/intra observer variability of manual brain tumor segmentation 14 15.

In the second study, we evaluated the results of our OPG segmentation and component classification method with a follow-up study. MR images of three patients with OPG were serially acquired at subsequent time intervals. The OPG and its three components were then manually segmented by an expert radiologist. For the automatic processing, we defined the first scan of every patient as the refer-
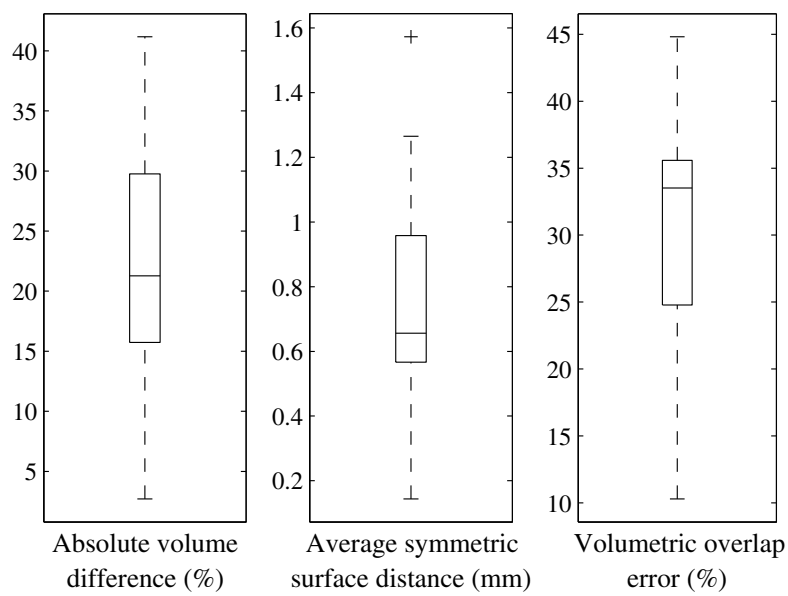

Fig. 2. Segmentation results summary for 25 cases ence scan and registered all subsequent scans to it. We then applied our method to each dataset, and computed the segmented OPG volume and that of its solid, enhancing, and cystic components (Sec. 2.4).

We computed the difference vector for every OPG component over time for both manual and automatic classification results. The difference vector consists of the volume differences between consecutive scans, and therefore represents the changes of the OPG component over the time for each patient.

Fig. 3 shows an illustrative example and the results the OPG automatic classification results as compared to the manual classification. We computed the correlation coefficients between the manual and automatic difference vectors. 


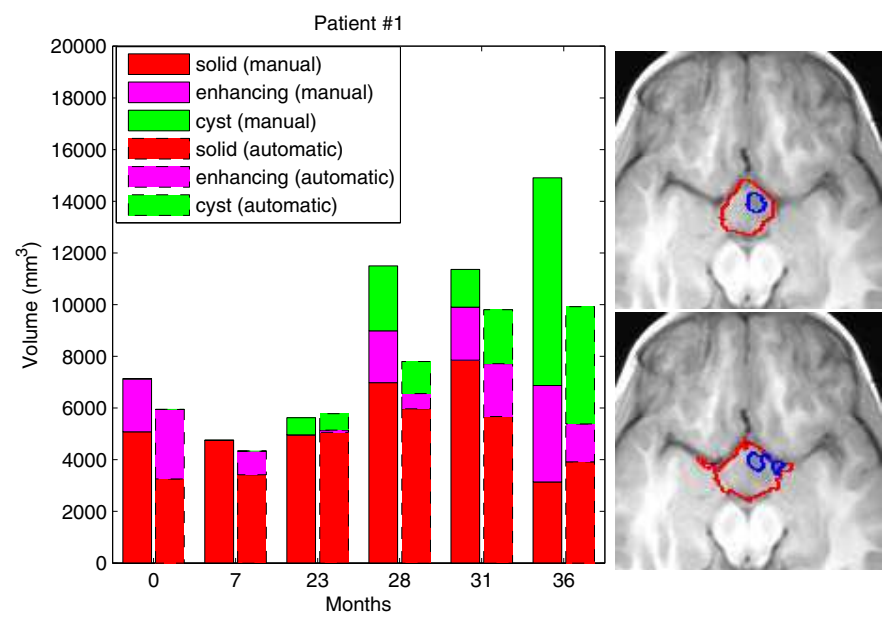

(a) (b)

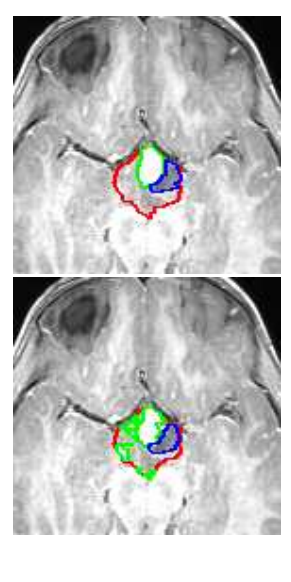

(c)

Fig. 3. Illustration of patient 1 follow-up study: (a) manual vs. automatic component classification chart; (b) and (c): ground truth (top) vs. our method (bottom) segmentation results on two patient 1 sample slices for months 23 (left) and 31 (right).

We also computed the same values for the standard Euclidean Distance (ED) classifier. Table 1 shows the results.

We conclude from Fig. 3 that our method successfully estimates the OPG volume progression. For example, the increase in the OPG volume of Patient 1, starting after 23 months, and

Table 1. SAM and ED correlation with ground truth

\begin{tabular}{|l|c|c|c|c|c|c|}
\hline & \multicolumn{2}{|c|}{ Patient 1 } & \multicolumn{2}{c|}{ Patient 2 } & \multicolumn{2}{c|}{ Patient 3 } \\
\hline Component & SAM & ED & SAM & ED & SAM & ED \\
\hline Solid & 0.778 & 0.085 & 0.597 & 0.487 & 0.161 & -0.468 \\
\hline Enhancing & 0.503 & 0.319 & 0.905 & 0.875 & 0.869 & 0.426 \\
\hline Cystic & 0.864 & 0.520 & N/A & N/A & 0.854 & 0.845 \\
\hline
\end{tabular}
the development of the enhancing component after 28 months, can be observed in both the manual and the automatic segmentation. These findings are an indicator for positive tumor progression, which may require altering the current patient treatment. From Table 1, we conclude that our method successfully estimates the OPG components progression. In addition, we found that our classification method outperforms the ED classifier, which relies on absolute grey-level intensity values.

\section{Conclusions}

We have presented a method for the automatic segmentation and component classification of OPGs from multi-spectral MRI. The paper makes three main contributions. First, our segmentation method uses a spatial a priori anatomical atlas to find the initial location of the OPG tumor. This is usually done manually via seed selection or by other means in existing segmentation methods. Second, our method classifies voxels according to the learned ratio between the pulse sequences, rather 
than by their absolute values. This yields a robust classification method that can handle gray-level intensity imaging variations. Third, we evaluated our method with a follow-up study on three patients, in addition to the standard measures of volume overlapping and surface distance. The study compares the relative volume progression of the OPG components at different times, and quantitatively supports the clinical findings. This constitutes a methodological improvement over the manual method currently used.

For future work, we are planning an extensive follow-up study. We plan to use the new ROI-based segmentation and SAM classification techniques for the automatic segmentation and classification of other types of brain tumors.

\section{References}

1. Huson, S.M., Hughes, R.A.: The Neurofibromatoses: a Pathogenetic and Clinical Overview. Chapman \& Hall, London (1994)

2. Binning, M.J., Liu, J.K., Kestle, J.R.W., Brockmeyer, D.L., Walker, M.L.: Optic pathway gliomas: a review. Neurosurgical Focus 23(5) (2007)

3. Liu, J., et al.: A system for brain tumor volume estimation via MR imaging and fuzzy connectedness. Comput. Med. Imag. Grap. 29(1), 21-34 (2005)

4. Corso, J.J., et al.: Efficient multilevel brain tumor segmentation with integrated bayesian model classification. IEEE T. Med. Imaging 27(5), 629-640 (2008)

5. Prastawa, M., et al.: Automatic brain tumor segmentation by subject specific modification of atlas priors. Acad. Radiol. 10, 1341-1348 (2003)

6. Smirniotopoulos, J.G.: The new WHO classification of brain tumors. Neuroimag. Clin. N. Am. 9(4), 595-613 (1999)

7. Lee, C.H., et al.: Segmenting brain tumor with conditional random fields and support vector machines. In: Proc. Int. Conf. Comput. Vision, Beijing, China, pp. 469-478 (October 2005)

8. Kaus, M., et al.: Automated segmentation of MRI of brain tumors. Radiology 218, 586-591 (2001)

9. Friston, K.J., Holmes, A.P., Ashburner, J.: Statistical Parametric Mapping (SPM) (1999), http://www.fil.ion.ucl.ac.uk/spm/

10. Cox, I.J., Hingorani, S.L.: Dynamic histogram warping of image pairs for constant image brightness. In: Int. Conf. on Image Proc., Washington, D.C, USA, vol. II, pp. 366-369. IEEE, Los Alamitos (October 1995)

11. Kay, S.: Fundamentals of statistical signal processing: detection theory. Prentice Hall, Englewood (1998)

12. Park, B., et al.: Classification of hyperspectral imagery for identifying fecal and ingesta contaminants. In: Proc. of SPIE, vol. 5271, pp. 118-127 (2003)

13. Laboratory of brain anatomical MRI. Johns Hopkins Medical Institute, http://cmrm.med.jhmi.edu/

14. Weltens, C., et al.: Interobserver variations in gross tumor volume delineation of brain tumors on computed tomography and impact of magnetic resonance imaging. Radiother. Oncol. 60, 49-59 (2001)

15. Wetzel, S.G., et al.: Relative cerebral blood volume measurements in intracranial mass lesions: interobserver and intraobserver reproducibility study. Radiology 224, 797-803 (2002)

16. Gerig, G., et al.: Valmet: A new tool for assessing and improving 3D object segmentation. In: Niessen, W.J., Viergever, M.A., et al. (eds.) MICCAI 2001. LNCS, vol. 2208, pp. 516-523. Springer, Heidelberg (2001) 